\title{
Evangelikalismus in Afrika
}

Evangelikalismus in Afrika ist ein schwieriges, wenn nicht gar unmöglich zu bestimmendes Arbeitsfeld, denn oft bleibt unklar wer oder was hierunter verstanden wird. Nimmt man etwa diverse Presseberichte als Grundlage, erscheint Evangelikalismus in Afrika eher als Bedrohungsszenario einer fremdgesteuerten und hochproblematischen Religiosität. So folgen Berichte in der FAZ oder ZEIT dem Narrativ des Films „God Loves Uganda“ und stellen das „AntiHomosexualitäts-Gesetz“ Ugandas als direkte Konsequenz amerikanischer evangelikaler Propaganda dar (Scheen 22. August 2014; Kemper 21. Februar 2013). ${ }^{1}$ In der NZZ werden Pastoren und Evangelisten aus dem Umkreis der sogenannten „Wohlstandskirchen“ („Prosperity Gospel“) als „evangelikale Führer“ charakterisiert (Signer 15. Januar 2016), und in der katholischen Zeitschrift Stimmen der Zeit sieht Katharina Hofer (2006) im Evangelikalismus ein „militantes Christentum“ heranwachsen. Meist geht es bei diesen und ähnlichen Berichten um genau genommen um pfingstliche Gruppen, aber die Bezeichnung „Evangelikalismus“ wird offenbar vorgezogen, weil sie einen Anschluss an die amerikanischen „Culture Wars“ erleichtert.

Sieht man sich dagegen in der internationalen akademischen Diskussion um, so konnte sich bislang kein derart umrissenes Forschungsfeld „Evangelikalismus“ etablieren. Überblickswerke zu pfingstlichen Kirchen finden sich fast immer unter dem Stichwort "Pentecostalism” (z.B. Kalu 2008; Lindhardt 2015) oder "Charismatics" (Asamoah-Gyadu 2005), und wenn breitere Begriffe dafür verwendet werden, rekurrieren Autoren auf Formulierungen wie "born-again Christianity" (Marshall 2009) oder einfach "new Christianity” (Gifford 2004). Wo hingegen die Bezeichnung “Evangelicals” gewählt wird, umreißt sie ein ungleich breiteres Feld, das von den etablierten protestantischen Kirchen über die Pfingstbewegung bis hin zu den sogenannten „AfrikanischUnabhängigen Kirchen“ reicht (Freston 2001; Ranger 2008). Diese Breite entspricht auch der Verwendung des Begriffs in Afrika. Nationale Kirchenräte, wie das “Evangelical Churches’ Fellowship of Ethiopia”, umfassen alle größeren protestantischen Kirchen, von Lutheranern und Mennoniten, bis hin zu Baptisten und den meisten Pfingstlern. Diese nationalen Organisationen sind wiederum in der Association of Evangelicals in Africa (AEA) zusammengeschlossen, die Mitglied in der World Evangelical Alliance ist und deren Glaubensbekenntnis teilt. Zugleich betreibt sie aber theologische Ausbildungsstätten wie die Nairobi Evangelical Graduate School of Theology (NEGST), deren Lehrkörper sowohl Absolventen aus etablierten Universitäten als auch aus evangelikalen Hochschulen umfasst.

Eine Erörterung des „Evangelikalismus“ in Afrika muss sich daher vor allem mit der Frage beschäftigen, ob und wie diese Kategorie auf das dortige evangelische Christentum abgebildet werden kann. Diese Frage soll anhand von drei Strömungen erörtert werden, die in verschiedener Weise mit dem „Evangelikalismus“ in Verbindung gebracht wurden: die Missionsbewegung des 19. Jahrhunderts, die etwas späteren Glaubensmissionen und Heiligungsbewegung, und die noch rezentere Pfingstbewegung. Dabei soll nicht nur der heuristische Wert der Kategorie des Evangelikalismus kritisch in den Blick genommen werden, sondern auch spezifische historische und kontextuelle Parameter herausgestellt werden, anhand derer die konfessionellen Dynamiken des

1 Eine angemessene Darstellung der komplexen Gemengelage aus Traditionsbezug, Politik, Religion und postkolonialen Identiätsdebatten im Umfeld des Uganda-Gesetzes findet sich dagegen bei Cheney (2013). 
evangelischen Christentums in Afrika besser verstanden werden können.

\section{Missionsbewegung und „Evangelikalismus“}

Die Bezeichnung „evangelical“ ist eng mit der aufkommenden protestantischen Missionsbewegung des langen 19. Jahrhunderts verbunden. Diese darf jedoch nicht in derselben spezifischen theologischen und kirchentrennenden Weise verstanden werden, wie dies für die „new evangelicals“ (Stanley 2013, S. 29) der Fall ist. Vielmehr steht „evangelical“ hier für die puritanischen und methodistischen Erweckungsbewegungen des 18. Jahrhunderts, deren Einfluss quer durch mehrere Kirchen ging.

Dies lässt sich bereits anhand der ersten Missionsgesellschaften zeigen. Die für Afrika sehr wichtige, bereits 1795 etablierte London Missionary Society (LMS), richtete sich in ihrem Gründungsaufruf sowohl an die „säuglingstaufenden evangelischen Dissenter“ („evangelical dissenters“), als auch an Anglikaner von „evangelischem Gefühl und einem lebendigen Eifer für die Sache Christi“ („of evangelical sentiments...“) (Lovett 1899, Bd. 1, S. 5, 16). Methodistische und kongregationalistische Kreise blieben zwar in der LMS federführend, doch als vier Jahre später mit der Gründung der Church Missionary Society (CMS) die etabliert-kirchliche Antwort folgte, war diese ihrerseits von der englischen Erweckungsbewegung inspiriert (Stock 1899). Es folgten zahlreiche weitere Gründungen im 19. Jahrhundert, die zumeist stärker an einzelnen Denominationen orientiert waren, sich jedoch in ihrem Missionsverständnis kaum von den früheren Gesellschaften unterschieden, wie die für Afrika wichtige Wesleyan Methodist Missionary Society (Findlay/Holdsworth 1922, Bd. 1, S. 36-55).

In Bezug auf diese Gruppen ist der englische Begriff “evangelical” mit dem Neologismus „evangelikal“ nur unzureichend wiedergegeben, denn sie sind eher analog zum Pietismus in Deutschland zu verstehen. Damit gehören sie zwar in die Genealogie des modernen Evangelikalismus, aber es ist auch deutlich, dass sie noch nicht mit den theologischen und kirchlichen Trennschärfen des heutigen Evangelikalismus-Begriffs gefasst werden dürfen, schon gar nicht in der Frage der „Heidenmission“. Auch die späteren hochkirchlichen Antworten auf das Aufkommen der Missionsgesellschaften, wie die Neuorientierung der Society of the Propagation of the Gospel in den 1830ern oder die Gründung der Universities' Mission to Central Africa (UMCA) im Jahr 1859, änderten daran nur wenig. Vielmehr nahmen sie den erwecklichen Impuls der „evangelicals“ in ihre stärker an kirchlichen Strukturen orientierten Missionsbemühungen auf.

Auf dem Kontinent verlief die Entwicklung der Missionsgesellschaften im Grund ähnlich. Den Anfang nahmen überkonfessionelle Missionsgesellschaften, die in deutlicher historischer Abhängigkeit zu den pietistischen Erweckungen standen, wie z. B. die Berliner Mission (1800/1824), die Baseler Mission (1815), oder die Rheinische Missionsgesellschaft (Gensichen 1976, S. 33-36). Wie in England folgte auch hier eine konfessionalistische Gegenbewegung ab den 1830ern, insbesondere in den lutherischen Kirchen Deutschlands. Hieraus entstanden Missionsgesellschaften wie die Leipziger Mission (1836), die Hermannsburger Mission (1849) oder die Deutsch-Ostafrikanische Missionsgesellschaft (1886). Auch sie unterschieden sich, bei allen sonstigen theologischen Differenzen, in ihrer Missionsrhetorik kaum von den überkonfessionellen, pietistisch geprägten Gesellschaften.

Ein Überblick über die Afrika-Arbeit der verschiedenen Missionen des 19. Jahrhunderts ist im 
Rahmen dieses Kapitels nicht zu leisten, zumal deren Rolle in der kolonialen Expansion Europas genauer Analysen bedarf (Altena 2003; Comaroff/Comaroff 1991; Harries 2007; Becher/Heyden 2000; Stoecker/van der Heyden 2005; Harries/Maxwell 2012; Porter 2002; 2006; Stanley 1990). Doch inwiefern lässt sich ihre Arbeit und Wirkung in Afrika als spezifisch „evangelikal“ verstehen? Historisch betrachtet, ist diese Frage aufgrund der bereits erwähnten Durchlässigkeit der Kategorie im 19. Jahrhundert eine anachronistische, doch wenn man mit Bebbingtons „Quadrilateral“ (1989, S. 2f.) eine stärker typologische Definition ansetzen möchte, so ließen sich durchaus deren vier Elemente auf die Missionsbewegung zurück projizieren (Bekehrung, Opfertodtheologie, Biblizismus, Aktionismus). Alle protestantischen Missionsgesellschaften setzten Bekehrungen als primäres Kriterium für den Erfolg ihrer Bemühungen an und betonten deren Notwendigkeit für jede Form der „Kulturbestrebung“ (Comaroff/Comaroff 1991, S. 198-251). Die Fokussierung auf den Opfertod Jesu als zentrale Bekehrungsbotschaft war ebenso beinahe universell, von Thomas Birch Freemans (1843) frühen Reisen zu den Ashanti bis hin zu den evangelistischen Bemühungen deutscher Missionare unter Muslimen in Ostafrika (Haustein 2016). Der Biblizismus der Missionsgesellschaften zeigt sich in ihrem Insistieren auf Vernakularsprachen in Bibelübersetzung und -unterricht (oft auch gegen koloniale Landessprachen), denn dieses gründete sich in einem Vertrauen auf die Kraft „des Wortes“ und dessen verlustfreie Übersetzbarkeit in die Begriffsfelder afrikanischer Sprachen. Diese sprachliche Aneignung afrikanischer Kosmologien für christliche Gottesvorstellungen schuf bemerkenswert hybride Konstruktionen, deren religiöse Sprengkraft sich nur mit einem literalistischen Urvertrauen in die normierende Kraft des Bibeltextes überdecken ließ (Comaroff/Comaroff 1991, S. 218). Der Aktionismus der Missionsbewegung lässt sich bereits an ihrer Verzahnung mit der Anti-Sklaverei-Bewegung ablesen. Die damit verbundenen Bestrebungen zur kulturellen und wirtschaftlichen „Hebung“ afrikanischer Gesellschaften führten Missionare oft weit über reine Evangelisierung hinaus. Bereits 1841 forderte Thomas Buxton ein Zusammengehen von Evangelisation mit „Schulmeister, Pflug und Spaten“ (1840, S. 511) und das Programm seiner Gesellschaft zur Förderung des Niger-Handels liest sich wie ein Vorläufer moderner Entwicklungshilfe (Buxton 1840, S. 1-10). Ein auf das Abstellen verschiedener Übelstände zielender Aktionismus blieb auch später ein zentraler und zunehmend professioneller Bestandteil der Mission, so dass sie als ein struktureller Vorläufer moderner NGOs angesehen werden können (Manji/O’Coill 2002).

\section{Glaubensmissionen, Heiligungsbewegung und die ostafrikanische Erweckung}

Mit Bebbingtons „Quadrilateral“ wäre also die gesamte protestantische Missionsbewegung und deren Kirchen zum Evangelikalismus zu zählen, was jedoch kaum der eingangs erwähnten populären Verwendung des Begriffs entspricht. Dagegen ließe sich mit einem stärker historisch gefassten Begriff des Evangelikalismus fragen, ob sich innerhalb der Missionsbewegung und der afrikanischen Kirchengeschichte des späten 19. und frühen 20. Jahrhunderts Strömungen oder Subkulturen des Protestantismus erkennen lassen, die enger mit der Genealogie der „new evangelicals“ verbunden sind, welche die heutige Verwendung des Begriffes „Evangelikalismus“ bestimmen (Suarsana in diesem Band).

Eine erste Gruppierung, an die man dabei denken könnte, sind die Glaubensmissionen. Damit werden die gegen Ende des 19. Jahrhunderts aufkommende Missionen bezeichnet, die als 
Gegenbewegung gegen die zunehmende Bürokratisierung und Konfessionalisierung der klassischen Gesellschaften entstanden. Gegründet auf dem „charismatischen Selbstrecht“ (Gensichen 1976, S. 41) eines Missionars bzw. einer „göttlichen Sendung“, banden sich diese Missionen an keine kirchliche Tradition oder dogmatische Position innerhalb des erweckten protestantischen Spektrums und verließen sich auf überkonfessionelle Unterstützerkreise. Die Bezeichnung „Glaubensmission“ gründet sich dabei auf dem beinahe universellen Prinzip, Missionare nicht zu entlohnen, sondern darauf zu vertrauen, dass Gott ihren materiellen Bedarf durch die „Hand seiner Kinder“ (Fiedler 1994, S. 33) decken werde. Die meisten dieser Gruppen zielten auf bislang noch nicht durch andere Missionen besetzte Gebiete in Afrika, oft verbunden mit der bereits auf C. F. Spittler und L. Krapf zurückgehenden Strategie einer ins Innere reichenden Kette von Missionsstationen. Ein weiterer Zweig der Glaubensmissionen in Afrika versuchte, sich durch Landwirtschaft und Handel selbst erhaltende Missionsstationen zu etablieren, was sich in der Praxis jedoch kaum als durchführbar erwies (Fiedler 1992, S. 96-100).

Die Arbeit der in Afrika tätigen Glaubensmissionen, wie der Africa Inland Mission, der Christian and Missionary Alliance oder der Sudan Interior Mission, war in den ersten Jahrzehnten kaum erfolgreich, denn viele zwar enthusiastische aber schlecht ausgerüstete Vorhaben scheiterten. Bis zum ersten Weltkrieg hatte kaum eine Glaubensmission mehr als nur ein Arbeitsfeld erschlossen (Fiedler 1992, S. 122). Die darauffolgenden wirtschaftlichen Schwierigkeiten der 1920er und 30er Jahre begrenzten ihre Möglichkeiten weiterhin. Dennoch lassen sich mehrere große Kirchen in Ländern Afrikas auf die Arbeit der Glaubensmissionen zurückführen (vgl. die etwas veralteten Zahlen in Fiedler 1992, S. 134-144), wobei die entscheidenden Phasen des Wachstums oft auf afrikanische Initiativen zurückzuführen sind. Ein interessantes Beispiel hierfür stellt Äthiopien dar, wo die von der Sudan Interior Mission gegründeten Versammlungen merklich dann wuchsen, als die Missionare wegen der italienischen Besatzung das Land verlassen mussten (Cotterell 1973, S. 101-106). Die Zentralität afrikanischer Initiativen ist auch für die Frage der evangelikalen Identität dieser Kirchen von Bedeutung. Denn lehrmäßige Abgrenzungen von anderen Kirchen dürften in schwierigen politischen Lagen und in Abwesenheit der Missionare eine untergeordnete Rolle gespielt haben, zumal wenn zuvor aufgrund der ubiquitären missionarischen Grenzabkommen ohnehin nur eine Art Kirche pro definierter Region etabliert worden war.

Ein theologisches Merkmal der Glaubensmissionen, das sie deutlich von ihren Vorgängern unterscheidet, ist ihre Verbindung und weitgehende Überlappung mit der Heiligungsbewegung. Die Heiligungsbewegung war eine Wiederaneignung der Wesleyanischen Lehre der christlichen Vervollkommnung, die sich ab Mitte des 19. Jahrhunderts in Amerika unter Methodisten, Baptisten und reformierten Kirchen ausbreitete (Dayton 1987, S. 63-84). Die daraus hervorgehende Lehre einer auf die Bekehrung folgenden ,zweiten Segnung“ fand auch in England Resonanz, insbesondere in den seit 1875 alljährlich einberufenen Versammlungen in Keswick, Nordengland, die vor allem von Anglikanern geleitet wurden. Sowohl die amerikanischen als auch die englischen Zweige der Heiligungsbewegung hatten mehrere Verbindungen zu den Glaubensmissionen und beeinflussten ihre Theologie und Frömmigkeitspraxis (Dayton 1987, S. 104-108; Fiedler 1992, S. 220-237).

In der Arbeit der Glaubensmissionen in Afrika war jedoch auch diese theologische Differenz kaum von Belang. Die Betonung eines „geheiligten“ Lebens nach der Bekehrung unterschied sich nicht 
wesentlich von den ethischen Forderungen der „Kulturmission“ und die Lehre des „zweiten Segens“ führte zu einem Aufschub der Taufe, was eher eine Angleichung der Glaubensmissionen an die Praxis der protestantischen Kirchen in Afrika bewirkte (Fiedler 1992, S. 245-251). Ein weiteres, in der Heiligungsbewegung fußendes Unterscheidungsmerkmal der Glaubensmissionen war ihre totale Ablehnung des Alkoholkonsums, die auch heute noch unter protestantischen Christen in Afrika weit verbreitet ist. Doch auch dieses Merkmal ist kein eindeutiges, denn die klassischen Wesleyanischen Missionen lehnten Alkoholkonsum ebenso strikt ab.

Zudem waren die Übergänge zwischen Heiligungsbewegung und den klassischen Missionen in Afrika eher fließend, was sich in der Ausbreitung von Erweckungsbewegungen zeigte. Sowohl die Pilkington-Erweckung im Uganda der 1890er als auch die hiermit lose verbundene ostafrikanische Erweckung der 1930er und 40er Jahre hatten zwar deutliche Wurzeln in der Heiligungsbewegung, aber gingen von Missionaren und Versammlungen der anglikanischen CMS aus (Sundkler/Steed 2000, S. 580-582; Ward/Wild-Wood 2012). Durch Literatur sowie internationale Reisen der involvierten Missionare und afrikanischen Prediger wurde die ostafrikanische Erweckung in den folgenden Jahrzehnten zu einem erfolgreichen afrikanischen „Exportprodukt“ in der englischsprachigen Welt und spielte noch im Lausanner Kongress von 1974 eine prominente Rolle (Stanley 2013, S. 81-85, 160, 168). Die hier abzulesende Vernetzung von CMS-Missionaren mit der sich neu gruppierenden evangelikalen Bewegung ist interessant, aber zugleich lief die Verbreitung der ostafrikanischen Erweckung über diese Kreise hinaus. Beides zeigt einmal mehr die Durchlässigkeit der Kategorie bzw. der innerevangelischen missionarischen Kontakte noch in den 1950ern und 1960ern und lädt zu einer gewissen Skepsis gegenüber der Reifizierung der Kategorie „Evangelikalismus“ selbst in dieser späten Zeit ein.

\section{Afrikanische Kirchen und Pfingstbewegung}

Mit dieser letzten Bemerkung soll freilich nicht in Abrede gestellt werden, dass bestimmte Kreise in der Nachkriegszeit genau diese Reifizierung als Programm betrieben, dessen globale Geschichte von Billy Grahams Evangelisationen bis hin zur Formierung der World Evangelical Fellowship und dem Lausanner Kongress - von Stanley (2013) im Detail nachgezeichnet worden ist. Stattdessen soll hier gezeigt werden, dass ausgerechnet diese Neuformierung evangelikaler Kreise von keiner größeren Bedeutung für die Christentumsgeschichte Afrikas war.

Billy Grahams autobiographischer Bericht über seine Afrikareise von 1960 macht dies exemplarisch deutlich (Graham 1997, S. 337-352). Trotz vergleichsweise großer Zuhörerzahlen war die „Ernte“ seiner Versammlungen geringer als anderswo, und abgesehen von einer Reise nach Südafrika im Jahr 1973 kehrte er nicht persönlich auf den Kontinent zurück, sondern überließ die Afrika-Arbeit seinem Sohn und anderen Mitarbeitern. Sein Resümee der Afrikareise fiel gespalten aus: einerseits sei der Kontinent mit der beginnenden Unabhängigkeit „reif für eine einmalige geistliche Erweckung“, aber andererseits bestehe die Gefahr eines „geistlichen Kampfes und sozialem Chaos, bei dem animistische und tribalistische Weisen mit allem Möglichen kollidieren, von Islam bis zu westlichem Konsum“ (Graham 1997, S. 350).

Diese etwas ratloses Bemerkungen verweisen ungewollt auf die eigentliche Dynamik der afrikanischen Christentumsgeschichte des 20. Jahrhunderts, nämlich auf die wachsende Unmöglichkeit, afrikanische Kirchen westlichen Missionen zu unterstellen, und die damit 
verbundene Entstehung eines hybriden und pluralen Christentums, das wesentliche Aspekte lokaler Kultur, Politik,religiöser Pluralität und sozialer Umstände mit globalen christlichen Einflüssen synthetisiert. Diese Dynamik entfaltete sich in unterschiedlicher Weise, doch für die Fragestellung dieses Kapitels ist insbesondere das Aufkommen der Pfingstbewegung von Interesse, da diese gelegentlich als Unterkategorie des Evangelikalismus (miss)verstanden wird.

Ein wichtiger theologischer Vorläufer der Pfingstbewegung war die Heiligungsbewegung, und ohne die mit ihr eng verbundenen Glaubensmissionen, ist auch die rasche Ausbreitung der frühen pfingstlichen Mission nicht zu erklären (Bergunder 2009). Ebenso wichtig war aber auch die Heilungsbewegung, deren Betonung einer übernatürlichen Heilung den Graben zu den Fundamentalisten vertiefte, der sich von nun an mitten durch das evangelikale Spektrum zog (Synan 2001, S. 75-78). Auch wenn ab den 1940er Jahren durch die National Association of Evangelicals ein gewisser Brückenschluss mit (vorwiegend den weißen) Teilen der Pfingstbewegung gelang, ist die Verhältnisbestimmung von Pfingstbewegung und Evangelikalismus nach wie vor umstritten (Cross 2014).

Die ersten pfingstlichen Missionare erreichten Afrika bereits wenige Monate nach dem Aufkommen der Azusa-Street-Erweckung von 1906, die oft als Beginn der Pfingstbewegung angesetzt wird (was nicht unproblematisch ist, Haustein/Maltese 2014, S. 26-27). Doch spricht wenig dafür, in diesen frühen Missionen den späteren Erfolg der afrikanischen Pfingstbewegung oder deren Identität zu verorten. Die Pfingstmissionen waren marginal, von Misserfolgen und Paternalismus gekennzeichnet, und bis auf wenige Ausnahmen wuchsen ihre Kirchen erst nach dem zweiten Weltkrieg in nennenswerter Weise (Anderson 2007, S. 149-190).

Das Wachstum der Pfingstbewegung in Afrika erklärt sich eher aus einer Reihe späterer Faktoren. Erstens bildeten sich recht schnell missionsunabhängige Pfingstkirchen, etwa durch frühzeitige Emanzipation (wie in Äthiopien, Haustein 2011b) oder die stetige Zurückdrängung missionarischer Einflüsse. Zugleich gründeten Afrikaner eine zunehmende Anzahl von Kirchen ohne Beteiligung westlicher Missionen, was seit der Unabhängigkeit afrikanischer Staaten immer mehr zum Normalmodus pfingstlicher Ausbreitung wurde. Die Pfingstbewegung weist mit dieser Ausbreitungsstruktur gewisse Ähnlichkeiten zu den sogenannten „Afrikanisch-Unabhängigen Kirchen“ (AUKs) auf, doch sollte sie nicht einfach mit letzteren zusammen geordnet werden. Eine übergreifende, typologische Diskussion von Pfingstbewegung und AUKs bleibt unbefriedigend (Anderson 2004, S. 103-106), was vor allem an der seit Sundkler (1948) durch Missionare geprägten Kategorie der AUKs selbst liegt, die historisch nicht oder kaum miteinander zusammenhängende Bewegungen unter derselben (begrifflich problematischen) Rubrik zusammenfasst. An zwei Stellen gab es allerdings konkrete historische Übergänge zwischen AUKs und der Pfingstbewegung: In Südafrika gingen die Zionistischen Kirchen aus einer frühen Spaltung zwischen Heilungsbewegung und Pfingstbewegung hervor (Anderson 2001, S. 93-124) und in Nigeria haben große Pfingstkirchen ihre Wurzeln in der Aladura-Bewegung (Quaas 2011).

Ein zweiter Faktor für das Wachstum der Pfingstbewegung seit dem zweiten Weltkrieg war ihre trotz oder gerade mit der stärkeren Unabhängigkeit einhergehende lose Vernetzung mit Pfingstkirchen und -theologien in den USA und Europa. Dies wurde vor allem durch einen neuen Typus der Mission bzw. Evangelisation ermöglicht, der nicht mehr an einer kontinuierlichen Präsenz oder missionarischen Dominanz, sondern an Großveranstaltungen und der medialen 
Verbreitung bestimmter Lehren interessiert war. Evangelisten wie William Branham, Gordon Lindsay, Oral Roberts, T. L. Osborn, und Reinhard Bonnke brachten seit den 1950er Jahren eine pfingstliche Wiederauflage der Heilungsbewegung hervor, die mit Kenneth Hagin und Kenneth Copeland in eine Betonung des persönlichen Glaubens zum Erlangen von Gesundheit, Erfolg und Reichtum überging, während Derek Prince Geistbesessenheit und Exorzismen in den Mittelpunkt seiner Verkündigung stellte. Nicht alle dieser Evangelisten reisten persönlich nach Afrika, doch ihre Lehren wurden breit und zeitnah durch Medien (Bücher, Zeitschriften und zunehmend audiovisuelle Medien) und persönliche Kontakte rezipiert. Für afrikanische Pfingstler war diese Art der Vernetzung ein attraktives Modell, denn es vermittelte globale Anschlüsse und das damit einhergehende Prestige, meist ohne direkte Einflussnahme der globalen Evangelisten auf die Kirchen selbst. Zudem boten ihre Sonderlehren eine gute Gelegenheit zur Profilierung gegenüber den älteren Pfingstkirchen, während sich ihre Massenveranstaltungen und Mediennutzung zur Nachahmung empfahl.

Die Rekombination pfingstlicher Sonderlehren im Wettbewerb der Kirchen untereinander steht im Zusammenhang mit einem dritten Faktor, der in der Forschung zur Pfingstbewegung breit ausgearbeitet wurde, nämlich der kontextuellen Adaptivität der Bewegung. Birgit Meyer (1999, 1998) hat darauf verwiesen, dass Pfingstler durch die Umdeutung traditioneller Kosmologien und Praktiken in christliche Dämonologie eine Brücke zwischen ländlicher „Vergangenheit“ und den Anforderungen der modernen, urbanen Lebensumstände schlagen. Sie inszenieren daher durch ihre Austreibungspraxis nicht nur den Bruch mit den Geistern der „alten Religion“, sondern betonen zugleich deren fortdauernde Wirksamkeit als stetig präsente Gefahr. Ruth Marshall $(2009,1995)$ hat gezeigt, wie die okkulte Ökonomie des Erfolgs in der nigerianischen Pfingstbewegung eine zur postkolonialen Turbulenz Nigerias alternative aber zugleich strukturparallele Vision von Politik und Subjektivierung erzeugt. David Maxwell (2006, 1998) verwies wiederum auf die Passung pfingstlicher Ethik zur neoliberalen Ökonomie in Zimbabwe, wogegen Päivi Hasu (2012) mit Blick auf Tansania die schicht- und ressourcenspezifische Handlungsrationalität verschiedener pfingstlicher Theologien und Praktiken herausgearbeitet hat. Die in diesen Beispielen erkennbare innovative und flexible (wenn auch oft kontroverse) Adaption an religiöse, politische und sozioökonomische Umstände unterstreicht die Alltagsrelevanz und Attraktivität pfingstlicher Kirchen in Afrika.

Die damit einhergehende wettbewerbsförmige Dynamik pfingstlicher Theologie und Frömmigkeitspraxis hat, viertens, auch die älteren Kirchen Afrikas in Form charismatischer Bewegungen erreicht und bezieht diese weitgehend in den pfingstlichen Diskurs mit ein. In Äthiopien etwa, wo der Bevölkerungsanteil der Protestanten in den letzten drei Jahrzehnten von fünf auf über zwanzig Prozent gestiegen ist, sind viele Sonntagsgottesdienste lutherischer Kirchen kaum von pfingstlichen zu unterscheiden. Unterschiede in Intensität und Art der Frömmigkeitspraxis verlaufen nicht mehr entlang denominationeller Demarkationen, sondern mitten durch diese hindurch (Haustein 2011b, 2011a). Auch in anderen Ländern Afrikas, wie etwa Kenia, Nigeria, oder Südafrika ist der Anteil der pfingstlich/charismatischen Christen bemerkenswert hoch (Pew 2006).

Das Aufkommen und die fortwährende Ausbreitung der Pfingstbewegung ist ohne Zweifel die wichtigste Entwicklung im afrikanischen Christentum der letzten Jahrzehnte. Doch ihre Einordnung 
in die Geschichte des Evangelikalismus ist nicht ohne Probleme. Die Pfingstbewegung lässt sich über die Heiligungsbewegung vielleicht als historischer Abzweig des Evangelikalismus verstehen, sie ist aber, wie bereits erwähnt, nicht einfach als Subkategorie des amerikanischen Evangelikalismus zu fassen. Zudem ist die afrikanische Pfingstbewegung aufgrund ihrer internen Pluralität, kontextuellen Adaptivität und Durchdringung vieler klassischer Kirchen kaum noch inhaltlich zu bestimmen. In der sich immer stärker spezialisierenden Forschung zu diesem Thema ist daher bereits der Punkt erreicht, wo einschlägige Sammelbände zwar ihre Vielseitigkeit deutlich herausstellen, aber eine zusammenfassende Synthese nicht mehr gut gelingt (Freeman 2012; Lindhardt 2015).

\section{"Evangelicals" in Afrika: Evangelisch oder Evangelikal?}

Die Geschichte des evangelischen Christentums in Afrika verdeutlicht also die heuristischen Grenzen der Kategorie „evangelikal“. Wenn man ein breites Konzept des Evangelikalismus wählt, das historisch im Pietismus beginnt und systematisch mit Bebbingtons Quadrilateral beschrieben wird, wären alle protestantischen Missionen in Afrika und die aus ihnen hervorgegangenen Kirchen als evangelikal zu bezeichnen, was auch am ehesten der Verwendung von "evangelical” in den kirchlichen Selbstbezeichnungen und Zusammenschlüssen Afrikas entspricht. Die Stärke dieses breiten Konzepts des Evangelikalismus besteht darin, dass es die historische Verwobenheit der Missions- und Erweckungsbewegungen deutlich herausstellt und es somit vor einer anachronistischen Übertragung der gegenwärtigen engeren Verwendung des Begriffs bewahrt. Diese breite Begriffsbestimmung im Sinne von „evangelisch“ steht freilich in Spannung zu der üblicherweise mit „evangelikal“ bezeichneten Differenzierung zwischen „etablierten“ protestantischen Kirchen und erweckten oder fundamentalistischen Gruppen der Gegenwart.

Wenn andererseits ein solcher, enger Begriff zur Anwendung kommen soll, so könnte man versuchen, die Glaubensmission und Heiligungsbewegung als Keimzellen des Evangelikalismus in Afrika anzusetzen. Jedoch blieben beide in Afrika vielerorts marginal, und wo größere Kirchen aus ihnen hervorgegangen sind, spielten weder die theologischen Abgrenzungen der Missionen eine wesentliche Rolle, noch sind überregionale Zusammenschlüsse dieser Kirchen entstanden, während es durchaus fließende Übergänge zu den übrigen evangelischen Kirchen und Missionen gab. Spätestens die Pfingstbewegung sprengt einen solchen eng gefassten Evangelikalismus-Begriff wieder auf, sowohl hinsichtlich der durch sie hervorgebrachten Theologien, Frömmigkeitsformen und kontextuellen Dynamiken, als auch der mit ihrer Verbreitung einhergehenden Verwischung zwischen „erweckten“ und „etablierten“ Kirchen.

Angesichts dieser Unmöglichkeit einer trennscharfen Bestimmung des afrikanischen Evangelikalismus bleibt abschließend festzustellen, dass der Begriff des Evangelikalismus für Afrika kaum analytischen Wert besitzt. Darum müssen in diesem Kapitel auch übergreifende Fragen zu Ausbreitung, regionalen Zentren, Spannungsfeldern und gesellschaftlichen Bedeutung des afrikanischen Evangelikalismus unbeantwortet bleiben. Selbst in stärker umrissenen Forschungsfeldern zum afrikanischen Christentum, wie etwa der Pfingstbewegung, setzt sich mittlerweile die Einsicht durch, dass man stärker als bislang zwischen verschiedenen Lehraussagen, Kirchen, Praktiken und lokalen Bedingungen unterscheiden muss, um die eigentlichen politischen und gesellschaftlichen Dynamiken zu verstehen, die hinter den eingangs erwähnten Problemfeldern von Sexualethik, finanzieller Ausbeutung und interreligiösen Spannungen stehen. 
Die Beschreibung und Analyse des afrikanischen Christentums sollte darum auf die Reifizierung theologischer Kategorien wie „Evangelikalismus“ verzichten, und zwar zugunsten einer präziseren Ausarbeitung der diachronen und synchronen Vernetzungen und Ideenverschiebungen, welche die fluide und komplexe Situation afrikanischer Kirchen in spezifischen Kontexten besser erschließt. (Bergunder 2009)..

\section{Weiterführende Literatur}

Anderson, Allan H. (2001): African Reformation. African Initiated Christianity in the 20th Century, Trenton, NJ: Africa World Press.

Hastings, Adrian (1994): The Church in Africa 1450-1950, Oxford: Oxford University Press.

Kalu, Ogbu U. (2008): African Pentecostalism. An Introduction, Oxford: Oxford University Press.

Lindhardt, Martin (Hrsg.) (2015): Pentecostalism in Africa. Presence and Impact of Pneumatic Christianity in Postcolonial Societies, Leiden: Brill.

Sundkler, Bengt/Steed, Christopher (2000): A History of the Church in Africa, Cambridge: Cambridge University Press.

\section{Literaturverzeichnis}

Altena, Torsten (2003): „Ein Häuflein Christen mitten in der Heidenwelt des dunklen Erdteils“. Zum Selbst- und Fremdverständnis protestantischer Missionare im kolonialen Afrika 18841918, München: Waxmann Verlag.

Anderson, Allan H. (2001): African Reformation. African Initiated Christianity in the 20th Century, Trenton, NJ: Africa World Press.

Anderson, Allan H. (2004): An Introduction to Pentecostalism, Cambridge: Cambridge University Press.

Anderson, Allan H. (2007): Spreading Fires. The Missionary Nature of Early Pentecostalism, London: SCM Press.

Asamoah-Gyadu, J. Kwabena (2005): African Charismatics. Current Developments within Independent Indigenous Pentecostalism in Ghana, Leiden: Brill.

Bebbington, David W. (1989): Evangelicalism in Modern Britain. A History from the 1730s to the 1980s, London: Routledge.

Becher, Jürgen/Heyden, Ulrich van der (Hrsg.) (2000): Mission und Gewalt. Der Umgang christlicher Missionen mit Gewalt und die Ausbreitung des Christentums in Afrika und Asien in der Zeit von 1792 bis 1918/19, Stuttgart: Franz Steiner.

Bergunder, Michael (2009): Der „Cultural Turn“ und die Erforschung der weltweiten Pfingstbewegung, in: Evangelische Theologie 69, S. 245-269.

Buxton, Thomas Fowell (1840): The African Slave Trade and Its Remedy, London: John Murray.

Cheney, Kristen (2013): Locating Neocolonialism, “Tradition,” and Human Rights in Uganda’s “Gay Death Penalty”, in: African Studies Review 55, S. 77-95.

Comaroff, Jean/Comaroff, John (1991): Of Revelation and Revolution, Bd. 1, Chicago, IL: University of Chicago Press.

Cotterell, F. Peter (1973): Born at Midnight, Chicago, IL: Moody Bible Institut.

Cross, Terry (2014): Sind Pfingstler evangelikale Christen? Eine Betrachtung der theologischen Unterschiede und Gemeinsamkeiten, in: Jörg Haustein/ Giovanni Maltese (Hrsg.), Handbuch pfingstliche und charismatische Theologie. Göttingen: Vandenhoeck \& Ruprecht, S.383-407.

Dayton, Donald W (1987): Theological Roots of Pentecostalism, Metuchen, NJ: Scarecrow Press.

Fiedler, Klaus (1992): Ganz auf Vertrauen. Geschichte und Kirchenverständnis der Glaubensmissionen, Giessen: Brunnen.

Fiedler, Klaus (1994): The Story of Faith Missions. From Hudson Taylor to Present Day Africa, Oxford: Regnum Books. 
Findlay, G.G./Holdsworth, W. W. (1922): The History of the Wesleyan Methodist Missionary Society in Five Volumes, London: Epworth Press.

Freeman, Dena (Hrsg.) (2012): Pentecostalism and Development. Churches, NGOs and Social Change in Africa, Basingstoke: Palgrave Macmillan.

Freeman, Thomas Birch (1843): Journal of Two Visits to the Kingdoms of Ashanti, in Western Africa, London: Wesleyan Methodist Mission Society.

Freston, Paul (2001): Evangelicals and Politics in Asia, Africa and Latin America, Cambridge: Cambridge University Press.

Gensichen, Hans-Werner (1976): Missionsgeschichte der neueren Zeit 3. Aufl., Göttingen: Vandenhoeck \& Ruprecht.

Gifford, Paul (2004): Ghana’s New Christianity. Pentecostalism in a Globalizing African Economy, Bloomington, IN: Indiana University Press.

Graham, Billy (1997): Just As I Am. The Autobiography of Billy Graham, Grand Rapids, Michigan: Zondervan.

Harries, Patrick (2007): Butterflies \& Barbarians. Swiss Missionaries \& Systems of Knowledge in South-East Africa, Oxford: James Currey.

Harries, Patrick/Maxwell, David (Hrsg.) (2012): The Spiritual in the Secular: Missionaries and Knowledge about Africa, Grand Rapids, MI: Eerdmans.

Hastings, Adrian (1994): The Church in Africa 1450-1950, Oxford: Oxford University Press.

Hasu, Päivi (2012): Responses to Socio-Economic Transformation in Tanzanian Pentecostal Christianity, in: Dena Freeman (Hrsg.), Pentecostalism and Development. Churches, NGOs, and Social Changes in Africa. Basingstoke: Palgrave Macmillan, S.67-86.

Haustein, Jörg (2011a): Charismatic Renewal, Denominational Tradition and the Transformation of Ethiopian Society, in: Evangelisches Missionswerk Deutschland (Hrsg.), Encounter Beyond Routine. Cultural Roots, Cultural Transition, Understanding of Faith and Cooperation in Development. Hamburg: EMW, S.45-52, abrufbar unter: http://www.emwd.de/fix/files/doku_5_encounter-beyond-routine2011.pdf, letzter Zugriff am 2.9.2011.

Haustein, Jörg (2011b): Writing Religious History. The Historiography of Ethiopian Pentecostalism, Wiesbaden: Harrassowitz.

Haustein, Jörg (2016): Provincializing Christian Missions. The Missionary Impasse in Engaging with Islam in German East Africa. Vortrag zum Workshop New Histories of Christianity in Southern Africa, University of Cambridge, 12. September 2016.

Haustein, Jörg/Maltese, Giovanni (2014): Pfingstliche und charismatische Theologie. Eine Einführung, in: diess. (Hrsg.), Handbuch pfingstliche und charismatische Theologie. Göttingen: Vandenhoeck \& Ruprecht, S.15-65.

Hofer, Katharina (2006): Entsteht in Afrika ein militantes Christentum? Zur öffentlichen Rolle des Evangelikalismus in Afrika südlich der Sahara, in: Stimmen der Zeit, S. 162-175.

Isichei, Elizabeth (1995): A History of Christianity in Africa. From Antiquity to the Present, London: SPCK.

Kalu, Ogbu U. (2008): African Pentecostalism. An Introduction, Oxford: Oxford University Press.

Kemper, Andrea Böhm und Anna (21. Februar 2013): Homophobie. Missionare des Hasses, in: Die Zeit, abrufbar unter: http://www.zeit.de/2013/09/Uganda-Missionare-

Homophobie/komplettansicht, letzter Zugriff am 22.1.2016.

Lindhardt, Martin (Hrsg.) (2015): Pentecostalism in Africa. Presence and Impact of Pneumatic Christianity in Postcolonial Societies, Leiden: Brill.

Lovett, Richard (1899): The History of the London Missionary Society 1795-1895, London: Henry Frowde.

Manji, Firoze/O’Coill, Carl (2002): The Missionary Position. NGOs and Development in Africa, in: International Affairs 78, S. 567-583.

Marshall, Ruth (1995): „God is Not a Democrat“. Pentecostalism and Democratisation in Nigeria, in: Paul Gifford (Hrsg.), The Christian Churches and the Democratisation of Africa. Leiden: Brill, S.239-260. 
Marshall, Ruth (2009): Political Spiritualities. The Pentecostal Revolution in Nigeria, Chicago: University of Chicago Press.

Maxwell, David (1998): „Delivered from the Spirit of Poverty?“ Pentecostalism, Prosperity and Modernity in Zimbabwe, in: Journal of Religion in Africa 28, S. 350-373.

Maxwell, David (2006): African Gifts of the Spirit. Pentecostalism \& the Rise of a Zimbabwean Transnational Religious Movement, Oxford: James Currey.

Meyer, Birgit (1998): „Make a Complete Break with the Past“. Memory and Post Colonial Modernity in Ghanian Pentecostalist Discourse, in: Journal of Religion in Africa 28, S. 316349.

Meyer, Birgit (1999): Translating the Devil. Religion and Modernity Among the Ewe in Ghana, Edinburgh: Edinburgh University Press.

Pew Forum on Religion \& Public Life (2006): Spirit and Power. A 10-Country Survey of Pentecostals, Washington, D.C.: Pew Research Center, abrufbar unter:

http://pewforum.org/uploadedfiles/Topics/Religious_Affiliation/Christian/Evangelical_Prote stant_Churches/pentecostals-08.pdf, letzter Zugriff am 10.2.2011.

Porter, Andrew (2002): Church History, History of Christianity, Religious History. Some Reflection on British Missionary Enterprise Since the Late Eighteenth Century, in: Church History 71, S. 555-584.

Porter, Andrew (2006): Missions and empire, c. 1873-1914, in: Sheridan Gilley/Brian Stanley (Hrsg.), The Cambridge History of Christianity. World Christianities, c. 1815-1914. Cambridge: Cambridge University Press, S.560-575.

Quaas, Anna D (2011): Transnationale Pfingstkirchen. Christ Apostolic Church und Redeemed Christian Church of God, Frankfurt am Main: Lembeck.

Ranger, Terrence (Hrsg.) (2008): Evangelical Christianity and Democracy in Africa, Oxford: Oxford University Press.

Scheen, Thomas (22. August 2014): Schwulen-Hass in Afrika Stellvertreterkrieg der Megakirchen, in: Frankfurter Allgemeine Zeitung, abrufbar unter: http://www.faz.net/aktuell/politik/ausland/afrika/radikale-evangelikale-in-afrika-predigenhass-auf-schwule-13108242-p2.html, letzter Zugriff am 22.1.2016.

Signer, David (15. Januar 2016): Evangelikale Führer: Freikirchen, Privatjets und Modernisierung in Afrika, in: Neue Zürcher Zeitung, abrufbar unter:

http://www.nzz.ch/international/aufgefallen/freikirchen-privatjets-und-modernisierung-inafrika-1.18677473, letzter Zugriff am 22.1.2016.

Stanley, Brian (1990): The Bible and the Flag. Protestant Missions and British Imperialism in the Nineteenth and Twentieth Centuries, Leicester: Apollos.

Stanley, Brian (2013): The Global Diffusion of Evangelicalism. The Age of Billy Graham and John Stott, Downers Grove, IL: IVP Academic.

Stock, Eugene (1899): The History of the Church Missionary Society, its Environment, its Men and its Work, London: Christian Missionary Society.

Stoecker, Holger/van der Heyden, Ulrich (Hrsg.) (2005): Mission und Macht im Wandel politischer Orientierungen. Europäische Missionsgesellschaften in politischen Spannungsfeldern in Afrika und Asien zwischen 1800-1945, Wiesbaden: Franz Steiner Verlag.

Sundkler, Bengt (1948): Bantu Prophets in South Africa, Cambridge: James Clarke \& Co.

Sundkler, Bengt/Steed, Christopher (2000): A History of the Church in Africa, Cambridge: Cambridge University Press.

Synan, Vinson (2001): In the Latter Days. The Outpouring of the Holy Spirit in the Twentieth Century, Fairfax, VA: Xulon Press.

Ward, Kevin/Wild-Wood, Emma (Hrsg.) (2012): The East African Revival, Aldershot: Ashgate.

Jörg Haustein ist Senior Lecturer in Religions in Africa an der School of Oriental and African Studies. Seine Forschungs- und Lehrschwerpunkte sind Pfingstbewegung weltweit und in Afrika (insbes. in Äthiopien), koloniale Religionsgeschichte Afrikas (insbes. in Deutsch- 
Ostafrika), sowie Religion und Entwicklungshilfe. 